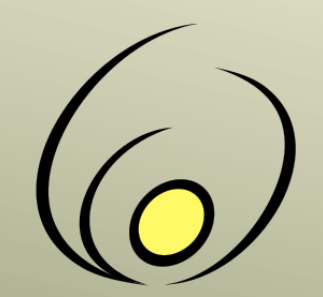

Fórum de Pró-Reitores de Extensão das Instituições Públicas de

Educação Superior Brasileiras

\section{Revista Brasileira de Extensão Universitária}

v. 12 , n. 3, p. 339-348, set.-dez. 2021

e-ISSN 2358-0399

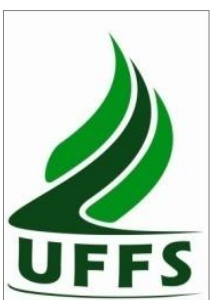

Content shared under Creative Commons Attribution 4.0 Licence CC-BY

\title{
Avaliação das condições higiênico-sanitárias e treinamento de manipuladores de alimentos em instituições públicas no município de Ipaumirim-CE
}

\author{
Naiara Menezes Bezerra1, Kássia Raffaela Roque Silva², Edsania Princelânia Xavier Nésio², \\ Lucélia Kátia de Lima3, Juliana Maria Guedes de Oliveira ${ }^{3}$
}

Resumo: As condições higiênico-sanitárias dos alimentos são de grande importância para a saúde pública, principalmente quando são oferecidos à coletividade, como em escolas. Dessa forma, o conhecimento acerca das Boas Práticas de Fabricação (BPF) na produção da alimentação escolar, além de essencial, é mandatório, pois é uma exigência legal. Este trabalho incluiu consultorias e atividades de assessoria, através de avaliações e capacitações nos serviços de alimentação em escolas da cidade de Ipaumirim - CE. Toda a metodologia utilizada foi baseada nas resoluções Resolução de Diretoria Colegiada (RDC) no 216 de 2004 e RDC no 275 de 2002 da Agência Nacional de Vigilância Sanitária (ANVISA). Primeiramente, foi realizada a aplicação de um checklist de condições higiênico-sanitárias nas escolas municipal (A) e estadual (B), seguida de avaliação das condições higiênicas dos manipuladores, por meio de análises microbiológicas. Por último, foi realizada uma capacitação desses manipuladores. A aplicação do checklist apontou um alto índice de inadequações. Nas análises microbiológicas feitas nos utensílios, a tábua de corte da escola A apresentou contaminação de Staphylococcus aureus. Nos demais utensílios e na escola B, não houve crescimento para os microrganismos analisados. Os resultados indicam condições estruturais precárias das escolas, evidenciando a necessidade de adequação às BPF. Contudo, os manipuladores apresentaram resistência tendo em vista a comparação das adequações antes e após a capacitação realizada, em especial na escola A, ficando clara a necessidade de serem realizadas mais ações e orientações sobre o cuidado higiênico-sanitário dos alimentos.

Palavras-chave: Merenda escolar; Higiene dos alimentos; Boas práticas; Capacitação

\section{Evaluation of hygienic-sanitary conditions and training of food handlers in public institutions in the municipality of Ipaumirim-CE}

Abstract: Hygienic and sanitary conditions of schools' food services have great importance for public health, especially when offered to the community, as in schools. Thus, the knowledge about Good Manufacturing Practices (GMP) in producing school meals, besides being essential, is mandatory as a legal requirement. This work included consulting and advisory activities through evaluations and training in food services in schools in the city of Ipaumirim, Ceará, Brazil. The methodology was based on resolutions RDC No. 216 of 2004 and RDC 2002 of ANVISA (Brazilian National Agency of Sanitary Vigilance). The first step was to apply a hygienic and sanitary conditions checklist in 2 schools (School A: district school and School B: state school). The second step was to evaluate the hygienic conditions of food handlers through microbiological analysis. The last step was the food handlers training. The checklist showed a high rate of inadequacies. The microbiological evaluation showed contamination by Staphylococcus aureus on the cutting board from school A. At school B, there was no growth for the analyzed microorganisms. The checklist results are compatible with the precarious structural conditions of schools, highlighting the need to adapt to GMP. However, the handlers showed resistance, given the comparison of the adaptations before and after the training, especially at school A, making it clear the need for further actions and guidance on hygienicsanitary food care.

Keywords: School meal, Food hygiene, Good practices, Capacitation

\section{Originais recebidos em}

06 de outubro de 2020

Aceito para publicação em

08 de setembro de 2021

1

Discente do Curso de Tecnologia em Alimentos - Instituto Federal de Educação, Ciência e Tecnologia da Paraíba IFPB/Campus Sousa

(autora para correspondência)

nayaramenezes ip@hotmail.com

2

Discentes do Curso de Tecnologia em Alimentos - IFPB/Campus Sousa

raffaelasilva64@gmail.com edsania97@gmail.com

3

Docentes e Pesquisadores do Curso de Tecnologia em Alimentos

luceliakatia@yahoo.com.br julianamgo@gmail.com 


\section{Introdução}

As escolas são cotadas como instituições base da organização da merenda, sendo que para algumas crianças e adolescentes essa refeição é a única completa do dia, isso acarretado por condições socioeconômicas. Por esse motivo é imprescindível que as mesmas ofereçam refeições nutritivas e que atendam aos critérios sanitários, sendo finalidades integrantes do Programa Nacional de Alimentação Escolar (PNAE), que atualmente é gerenciado pelo Fundo Nacional de Desenvolvimento da Educação (FNDE) do Ministério da Educação. O PNAE é considerado uma estratégia de promoção da Segurança Alimentar e Nutricional (SAN) (Conselho Nacional de Segurança Alimentar e Nutricional [CONSEA], 2007).

Muitas escolas oferecem refeições sem nenhum preparo adequado, tanto em termos técnicos quanto operacionais. Como os alimentos são passíveis de contaminação, a falta de cuidado dos manipuladores e o não cumprimento das Boas Práticas de Fabricação (BPF) propiciam a multiplicação de microrganismos transmissores de doenças (Silva et al., 2012b). No Brasil, segundo o Ministério da Saúde (2018) os surtos de doenças transmitidas por alimentos (DTA), entre os anos de 2000 a 2017, foram cerca de 8,6\% em creches e escolas. As crianças estão mais susceptíveis as DTA, por terem o sistema imunológico ainda em desenvolvimento, com menos capacidade de combater infecções (Lopes et al., 2015).

Dessa forma, é essencial o conhecimento acerca das BPF na produção da alimentação escolar. No processo produtivo das refeições, a conservação dos alimentos é a maior limitante, em função de sua acentuada perecibilidade, gerando potencial transmissão de microrganismos patogênicos. Com isso, o cumprimento das BPF é determinante para evitar os riscos de contaminação microbiana (Pereira \& Zanardo, 2020).

A segurança alimentar dos alimentos, por meio do desenvolvimento de Sistemas de Gestão e Controle de Qualidade e treinamentos dos colaboradores, cria, assim, um diferencial na área da indústria (Vasques \& Madrona, 2016). Neste sentido, programas devem ser criados para treinamentos de manipuladores de alimentos visando proteger a saúde dos consumidores (Silva \& Almeida, 2011).

Os programas de nutrição escolar, além de garantir uma alimentação segura para os alunos, devem implementar programas que garantam as condições higiênico-sanitárias para o alimento manipulado e o ambiente, bem como a saúde do manipulador, uma vez que esse pode ser um propagador de DTA, através de treinamento dos colaboradores, e uma instalação adequada para o manuseio de alimentos e matérias-primas de procedência segura.

A extensão universitária visa a levar o desenvolvimento e a aplicação de pesquisa e ensino realizados em seus departamentos acadêmicos, buscando modificar realidades e melhorar a qualidade de vida das populações assistidas. Por outro lado, abre a convivência e a interação com as comunidades e, no convívio, novos conhecimentos são descobertos e situações diferentes daquelas vivenciadas na área acadêmica acontecem (Moura et al., 2001).

Tais informações poderão contribuir para promover as políticas públicas, no sentido de estabelecer melhorias na execução do PNAE, a fim de garantir a produção de refeições adequadas e saudáveis, e proteger a saúde dos alunos (Gomes et al., 2012). Neste sentido, programas devem ser criados para capacitação de manipuladores de alimentos, visando proteger a saúde das crianças e adolescentes (Silva \& Almeida, 2011).

O presente estudo teve como objetivo desenvolver atividades de consultoria nas instituições de ensino municipal e estadual, através de avaliações e capacitação nos serviços de alimentação na cidade de Ipaumirim - CE. 


\section{Metodologia}

Nessa publicação estão contempladas as ações desenvolvidas na vigência do projeto de extensão intitulado "Avaliação das condições higiênico-sanitárias e treinamento de manipuladores dos alimentos em instituições públicas no município de Ipaumirim-CE", a fim de realizar uma intervenção social em duas instituições de ensino, uma de ensino médio e a outra de ensino fundamental, com duração de sete meses.

O projeto de extensão a partir do qual foi feito a confecção do presente artigo foi submetido e aprovado ao Comitê de Ética em Pesquisa do Instituto Federal da Paraíba, no ano de 2019, sob $n^{\circ}$ CAAE 13464919.3.0000.5185. A metodologia utilizada foi baseada nas resoluções da ANVISA RDC $n^{\circ} 216$ de 15 de setembro de 2004; RDC n 275 de outubro de 2002; American Public Health Association (2001) e métodos de análise de coliformes; Escherichia coli; Staphylococcus aureus.

Na primeira etapa do projeto, as atividades foram efetuadas na forma de pesquisa bibliográfica e roda de conversa entre os discentes, orientador e coorientador. A abordagem foi direcionada às temáticas que seriam enfatizadas durante as visitas, tais como: doenças transmitidas por alimentos (DTA), microrganismos, contaminação cruzada, boas práticas de manipulação (BPF) e conservação dos alimentos.

\section{Etapa 1- Aplicação da lista de verificação}

Inicialmente, foi realizada a averiguação através da lista de verificação, pelas as estudantes do curso de tecnologia em alimentos, capacitadas de acordo com a Resolução $n^{\circ} 216$, que dispõe os regulamentos de BPF para os serviços de alimentação (Agência Nacional de Vigilância Sanitária (ANVISA), RDC n 216, de 15 de setembro de 2004). Os serviços de alimentação de ambas as escolas ofertavam duas refeições ao longo do dia em dois turnos distintos, pela manhã e à tarde.

Nessa lista de verificação foram avaliadas: as condições de edificação, instalações internas e externas; as condições de higiene dos equipamentos, móveis e utensílios, e ambientes de produção. Além disso, foram avaliados o controle de pragas e vetores, abastecimento de água, manejo dos resíduos, higiene e saúde dos manipuladores, e como eram obtidas as matérias-primas e realizado o preparo, armazenamento e transporte dos alimentos. Outro fator observado foi a exposição ao consumo do alimento preparado, documentação e responsável técnico.

O índice de conformidade (\%) foi calculado conforme a seguinte equação:

Índice de conformidade $(\%)=\frac{\text { Total de SIM }}{\text { Total de itens- } N A} \times 100$

Onde: Total de SIM: número de itens conformes

Total de itens: Quantidades de itens avaliados

NA: São os itens em que não se aplica a avaliação de conformidade.

Para avaliação dos resultados, foram utilizados os parâmetros indicados pela RDC n ${ }^{\circ} 275$ de outubro de 2002, que classifica a conformidade de acordo com o percentual de atendimento aos itens: grupo 1 (76-100\%), grupo 2 (51-75\%) e grupo 3 (abaixo de 50\%) (Agência Nacional de Vigilância Sanitária, Brasil, RDC n²75, de 21 de outubro de 2002).

\section{Etapa 2- Realização de análises microbiológicas}

A execução das análises microbiológicas foi realizada no laboratório de microbiologia do Instituto Federal da Paraíba - IFPB - Campus Sousa. Tais análises foram realizadas com consentimento dos manipuladores e direção 
das escolas, bem como a aprovação do comitê de ética do Instituto Federal da Paraíba. Essas análises foram realizadas em amostras coletadas das superfícies dos utensílios, duas da escola A e duas da escola B, e das mãos dos manipuladores, sendo duas manipuladoras da escola $A$ e uma da B. O método utilizado foi o estabelecido pela American Public Health Association (2001), realizando o esfregaço das superfícies com Swab. As coletas das amostras ocorreram logo após a produção das refeições e o procedimento padrão de limpeza e/ou higienização da cozinha. As superfícies coletadas foram a tábua de corte, a faca mais utilizada, e as mãos das manipuladoras. O resultado foi expresso em Unidades Formadoras de Colônias (UFC) por superfície. Foram analisadas a presença e/ou ausência de bactérias como Escherichia coli, coliformes à $35^{\circ} \mathrm{C} \mathrm{e} 45^{\circ} \mathrm{C}$, e a contagem padrão em placas para Staphylococcus aureus. Ambas as análises são indicadores de contaminação por hábitos de higiene inadequados.

A análise de coliformes foi realizada pela metodologia de colimetria com a técnica do número mais provável, em duas etapas: o teste presuntivo, verificando-se a existência de coliformes através da fermentação da lactose com produção de gás, e o teste confirmativo, que permite observar se os coliformes da amostra são totais ou fecais, o primeiro tipo em incubação a $35^{\circ} \mathrm{C}$ por $48 \mathrm{~h}$, e o segundo a $45^{\circ} \mathrm{C}$ por $48 \mathrm{~h}$. Nessa análise foi usada os meios de cultura: Caldo Lauril Sulfato Triptose e Caldo Verde Brilhante Bile Lactose.

A análise de Staphylococcus aureus, com método de contagem direta em placa, com a inoculação 0,1 mL de cada diluição na superfície da placa e espalhada com alça de Drigalski, foi incubada em posição invertida a $37^{\circ} \mathrm{C}$ por 48 h. O meio de cultura utilizado foi Agar Baird-Parker (BP).

A análise de Escherichia coli, com método de contagem direta em placa, também seguiu como a abordagem anterior, com inoculação de $0,1 \mathrm{~mL}$ das diluições nas placas e a técnica de estrias múltiplas, com auxílio da alça, e foi incubada invertida a $35^{\circ} \mathrm{C}$ durante $24 \mathrm{~h}$. O meio de cultura utilizado foi o BD BEM Agar, modificado. Foram coletadas sete amostras para realização das análises em triplicatas. Todo o processo de coleta foi feito com Swab esterilizado acondicionado e transportado em meio Stuart ${ }^{\circledR}$, para conservar as células durante a viagem. Com auxílio de cooler e blocos de gelo, as amostras foram encaminhadas imediatamente para o laboratório. Primeiramente, foi feita a análise de coliformes totais, Staphylococcus aureus e Escherichia coli, nesta ordem.

\section{Etapa 3- Treinamento para os manipuladores}

Para o desenvolvimento do treinamento, foi feito uma revisão bibliográfica incluindo a RDC $n^{\circ} 216$ (ANVISA, RDC $\left.n^{\circ} 216,15.09 .2004\right)$, artigos, dissertações de mestrado sobre tal legislação, e a cartilha sobre boas práticas para serviços de alimentação da ANVISA e temas como: DTA, contaminação cruzada, riscos físicos, químicos e biológicos tanto para o ambiente, alimento e manipulador e sobre a conservação dos alimentos, com duração de 40 minutos. Em seguida, foram elaborados jogos interativos, folder, slidese apostilas e as demais atividades de capacitação, com duração prevista de 60 minutos. O treinamento foi realizado em um auditório no CRAS na cidade de Ipaumirim- $\mathrm{CE}$, com a participação de três manipuladoras das duas escolas.

O treinamento foi conduzido pelas três alunas universitárias e auxiliado por uma das orientadoras. Inicialmente, foi feita uma breve apresentação das palestrantes e, em seguida, foi conduzida a apresentação. A primeira palestrante abordou os assuntos: DTA, contaminação cruzada e riscos físicos e químicos; já a segunda, deu sequência aos riscos biológicos com o alimento e do manipulado com o alimento e higienização das mãos; e a terceira abordou sobre saúde dos manipuladores, armazenamento dos alimentos e técnicas de conservação, como a higienização de frutas e hortaliças, sobre temperaturas de conservação, dicas para o uso correto de descongelamento de carnes e produtos cárneos. Após essa abordagem, abriu-se tempo para dúvidas e relatos de experiência das manipuladoras, pelos quais elas abordaram dificuldades em relação à falta de materiais, de orientação, e tiraram dúvidas sobre a melhor maneira de conduzir os conhecimentos adquiridos. 
Foi planejado também um coffee break em ambiente adequado, e todos os equipamentos necessários para que as manipuladoras pudessem desfrutar de todas as informações com conforto. O treinamento teve duração de três horas, incluindo: palestra, jogos interativos, dúvidas e relatos de experiências. Os assuntos abordados foram relacionados à contaminação dos alimentos e boas práticas; os jogos interativos foram realizados com tinta guache, com o intuito de que os próprios manipuladores pudessem observar como realmente lavam as mãos, avaliando se está certo ou errado; as dúvidas e relatos de experiência foi uma oportunidade aberta para que elas ficassem à vontade para falar e elaborar questionamentos sobre as boas práticas. Ao final, as participantes receberam certificado de participação.

\section{Resultados e Discussão}

\section{Aplicação da lista de verificação}

Ao analisar os itens da lista de verificação das escolas, pode-se observar que ambas se classificaram no grupo 3, de alto risco (0 - 50\%), apresentando um alto índice de inadequações. Tais resultados são semelhantes ao estudo realizado por Lopes et al. (2015), ao avaliar as boas práticas em escolas públicas no município de Bayeux-PB.

As escolas possuem um responsável técnico nutricionista para as atividades de capacitação dos manipuladores e nutrição escolar, vinculado à prefeitura na escola $A$, e ao estado na escola B. Porém não estão acontecendo as capacitações periódicas aos manipuladores, evidenciando as irregularidades em diversas etapas do processo produtivo.

Na primeira visita foram analisadas todas as atividades desenvolvidas pelos manipuladores, sem nenhuma interferência por parte dos extensionistas, observando apenas a forma como elas usavam os EPI, a forma de higienização, e a exposição aos riscos do ambiente. A segunda visita ocorreu após o treinamento, quando foram observadas algumas mudanças positivas, por exemplo, na maneira de lavar as mãos.

Foi observado que os manipuladores não apresentavam um uniforme padrão, utilizando vestuários do cotidiano, como short ou calças tipo legging com cores inadequadas, e sem o uso de toucas. Resultados semelhantes foram encontrados por Silva et al. (2012a), ao avaliar as boas práticas em escolas do município de Castanhal-PA. A higiene pessoal do manipulador é de extrema importância, sendo preconizadas vestimentas adequadas, que devem ser mantidas limpas, e os adornos devem ser retirados durante a manipulação dos alimentos (ANVISA, RDC $n^{\circ} 216,15.09 .2004$ ). Foi observado também que os manipuladores não usavam sapatos fechados e antiderrapantes, que são modelos de calçados que deveriam ser utilizados no ambiente de processamento de alimentos. Segundo Lopes et al. (2015), a falta de utilização do calçado adequado durante a manipulação dos alimentos, além de ser anti-higiênico, também pode trazer riscos de acidentes para os manipuladores.

Ambas as escolas apresentaram várias irregularidades comuns nas instalações, como: objetos em desuso; insetos; espaços insuficientes para a produção e expedição; pisos de material de cor escura, não antiderrapante e em estado de conservação inadequado; paredes que não apresentam cores claras, e que possuem algumas infiltrações. As portas e janelas encontravam-se com poeira e sem telas para impedir a invasão de roedores e insetos. Além disso, a ventilação era insuficiente. Problemas nas instalações detectados no presente estudo, foram semelhantes aos observados por Lopes et al. (2015) e Silva et al. (2012b), em unidades de alimentação e nutrição de escolas. A Agência Nacional de Vigilância Sanitária (ANVISA) preconiza, na RDC n 216 de 2004, que as instalações físicas como piso, parede, teto, bancadas, portas e janelas devem possuir revestimento liso, impermeável e lavável, que devem ser mantidos íntegros, conservados, livres de rachaduras, trincas, goteiras, vazamentos, infiltrações, bolores, descascamentos, dentre outros. 
No quesito higienização dos equipamentos, foi observado um aspecto positivo, o fogão era higienizado após cada refeição, o mesmo com relação às bancadas. No entanto, o fogão apresentou danos, pois grande parte da estrutura estava enferrujada. Os utensílios eram guardados em prateleiras abertas, fator negativo, em razão da exposição a agentes biológicos.

Em relação ao controle de pragas e vetores, ambas as escolas afirmaram que a dedetização era realizada semestralmente, mas foram observadas teias de aranhas e moscas, a mesma situação evidenciada por Lopes et al. (2015). As duas não apresentavam proteção das luminárias, fato este que pode provar um risco físico, uma vez que, acontecendo algum acidente, pode cair material sobre o alimento, pondo em risco a saúde dos estudantes.

Os reservatórios de água são higienizados semestralmente, mas não apresentaram registros documentais na escola $A$, já na $B$ eles não souberam informar, e não tinham conhecimento da higienização do mesmo. Foi observado a falta de instalações exclusivas para lavagem e desinfecção das mãos. Com relação às instalações sanitárias, apenas a escola A disponibilizava sanitários exclusivos para os colaboradores.

Quanto ao requisito manuseio do lixo, as cozinhas encontram-se em conformidade, uma vez que elas dispõem de lixeiras de fácil limpeza, e o lixo é retirado diariamente, durante as trocas de turnos. Os produtos de limpeza eram armazenados em ambiente separado, e a higienização do ambiente era feita ao final de cada expediente.

Em relação ao grupo dos alimentos, ambas as instituições apresentaram situações irregulares, destacando-se as seguintes: a maneira incorreta de descongelar os produtos cárneos, uma vez que muitos produtos são deixados à temperatura ambiente por longos períodos para o descongelamento e, em seguida, usam-se sacolas plásticas que já haviam sido utilizadas para armazenar tais produtos descongelados. Observou-se também ausência de controle de temperatura nas frutas e verduras. Foi verificada a higienização inadequada dos produtos hortifrutigranjeiros, sem retirar devidamente os contaminantes microbiológicos, por meio da imersão em 15 minutos na água clorada.

O manuseio e a conservação adequada dos alimentos contribuem consideravelmente para a garantia de um alimento seguro e de qualidade por mais tempo (Silva et al., 2012b). O armazenamento dos produtos ocorreu em um local separado da área de preparação dos alimentos, respeitando o método PEPS (Primeiro que entra, Primeiro que sai) e ambas têm nas suas instalações freezer para o armazenamento de produtos cárneos, e outro para as frutas e hortaliças.

No requisito documentação, quanto aos Procedimentos Operacionais Padrão (POP) que a legislação enfatiza, os registros de higienização, e o manual de boas práticas, as duas instituições não apresentaram tais documentos, preconizados pelas resoluções RDC $n^{\circ} 275$ e RDC $n^{\circ} 216$, ambas da ANVISA. Este fato negativo compromete a integridade e qualidade dessas cozinhas, por não obedecerem a um padrão e não terem documentos e registros para recorrer no surgimento de dúvidas.

\section{Análises microbiológicas}

Os resultados observados demostram (Tabelas 1 e 2) que, das amostras apresentadas das superfícies, apenas a tábua de corte da escola A apresentou contaminação por Staphylococcus aureus, para os demais utensílios e a escola B não houve crescimento dos microrganismos analisados.

A contaminação presente na tábua que era de plástico pode ser justificada pelo fato desse utensílio estar em ambiente inapropriado e com grande possibilidade de contaminação, pois encontrava-se exposta e ao contato dos manipuladores, mesmo não sendo utilizado naquele momento. A mesma encontrava-se em condições de conservação mais precárias, mesmo o material sendo de plástico ainda pode manter biofilmes que servem de proliferação bacteriana se não obteve boa higienização. Uma alternativa é a utilização de água quente para 
auxiliar na limpeza. Resultados superiores de contaminação foram encontrados por Vila et al. (2014) ao avaliar as condições higiênico-sanitárias de cozinhas de escolas no Rio Grande do Sul, com 33,3\% das amostras com não conformidades em relação às bactérias.

Os resultados das mãos dos manipuladores (Tabela 2) mostraram contaminação dos dois colaboradores da escola $A$, ambos os resultados em nível de $2,0 \cdot 10^{2}$. Esse resultado pode ser explicado por vários fatores: ausência de uniforme e equipamentos de proteção individual, ausência de pias exclusivas, inexistência de sabonete líquido e antisséptico para adequada higienização das mãos, e também falta de capacitação e instruções sobre a importância das boas práticas na produção de alimentos. Os manipuladores de alimentos são importantes fontes veiculadoras de Staphylococcus aureus, pelo fato da maioria ser portadora assintomática. A presença desse microrganismo é um indicativo de condições higiênico-sanitárias insatisfatórias e interpretada como indicativo de contaminação a partir da pele, boca e/ou aparelho respiratório dos manipuladores de alimentos (Machado et al., 2009; Pedrosa et al., 2015). Esse microrganismo apresenta crescimento nas faixas de 7 a $47,8^{\circ} \mathrm{C}$, e produzem enterotoxinas entre 10 e $46^{\circ} \mathrm{C}$. Sua toxina é capaz de proliferar em temperatura ambiente (Franco \& Landgraf, 2008; Pedrosa et al., 2015) Tais toxinas são termoestáveis a $100^{\circ} \mathrm{C}$ por até 30 minutos, e possuem resistência à hidrólise pelas enzimas gástricas e jejuno. Desta maneira, se o produto alimentar estiver contaminado com o estafilococo produtor de enterotoxinas, e as toxinas tenham sido produzidas em grande quantidade, nem mesmo o aquecimento em elevadas temperaturas e a exposição a ácidos gástricos serão suficientes para evitar a intoxicação (Murray et al., 2014).

Tabela 1. Resultados das análises microbiológicas dos utensílios das escolas A e B.

\begin{tabular}{|c|c|c|c|c|}
\hline \multirow{3}{*}{$\begin{array}{c}\text { Análises } \\
\text { microbiológicas }\end{array}$} & \multicolumn{4}{|c|}{ Utensílios } \\
\hline & \multicolumn{2}{|r|}{ Escola A } & \multicolumn{2}{|r|}{ Escola B } \\
\hline & Faca & Tábua de corte & Faca & Tábua de corte \\
\hline Coliformes a $45^{\circ}$ & NO & NO & NO & NO \\
\hline Coliformes a $35^{\circ}$ & NO & NO & NO & NO \\
\hline Escherichia coli $\left(\mathrm{UFC} / \mathrm{cm}^{2}\right)$ & NO & NO & NO & NO \\
\hline Staphylococcus aureus (UFC/cm²) & NO & $>1,1 \cdot 10^{2}$ & NO & NO \\
\hline
\end{tabular}

Fonte: autoria própria (2020). NO: Não observado.

Tabela 2. Resultados das análises microbiológicas dos manipuladores das escolas.

\begin{tabular}{|c|c|c|c|}
\hline \multirow{2}{*}{$\begin{array}{c}\text { Análises } \\
\text { microbiológicas }\end{array}$} & \multicolumn{3}{|c|}{ Manipuladores } \\
\hline & $\begin{array}{c}\text { Escola A } \\
1\end{array}$ & $\begin{array}{c}\text { Escola A } \\
2\end{array}$ & $\begin{array}{c}\text { Escola B } \\
3\end{array}$ \\
\hline Coliformes a $45^{\circ}$ & NO & NO & NO \\
\hline Coliformes a $35^{\circ}$ & NO & NO & NO \\
\hline Escherichia coli (UFC/ $\mathrm{cm}^{2}$ ) & NO & NO & NO \\
\hline Staphylococcus aureus (UFC/ $\mathrm{cm}^{2}$ ) & $>2,0 \cdot 10^{2}$ & $>2,0 \cdot 10^{2}$ & NO \\
\hline
\end{tabular}


Com os resultados encontrados no presente estudo, foi observado que a escola B obteve bons resultados, uma vez que as amostras de utensílios e manipulador não apresentaram crescimento microbiano, fator importante na qualidade dos alimentos servidos; ainda sim, precisava de melhorias na sua estrutura física e na higiene e cuidados do manipulador.

Já na escola A, foi perceptível a contaminação da tábua de corte e da faca, com valores elevados, e das mãos dos manipuladores por $S$. aureus, ficando ainda mais evidente que a etapa de desinfecção não era realizada corretamente, tanto dos utensílios, como dos manipuladores. Foi observado que os utensílios eram submetidos a lavagem apenas para a retirada da matéria orgânica, e os vários requisitos de higiene do manipulador não eram atendidos, como observado na lista e verificação de boas práticas.

\section{Treinamento para os manipuladores}

O treinamento trouxe resultados para a escola B, observando-se após a capacitação algumas melhoras no manuseio dos alimentos e na higienização das mãos. Notou-se maior atenção à maneira correta de lavagem das mãos e preparo dos alimentos servidos. Já na escola A não houve uma absorção do conteúdo como o esperado, pois não apresentaram melhorias em nenhum aspecto avaliado antes do treinamento, notando que as manipuladoras apresentaram resistência a mudanças, pelo o fato das duas manipuladoras apresentarem um tempo maior de serviço e, com isso, acharem que tais sugestões dadas não iriam influenciar diretamente na manipulação. Tais observações foram enfatizadas na última visita após a aplicação do treinamento e notada essas afirmações.

Considerando o observado, ressalta-se que o manipulador precisa entender a sua responsabilidade e importância no serviço de alimentação, para assim garantir a segurança alimentar dos consumidores, sendo o treinamento o ponto de partida (Dias \& Santos, 2017). Sendo assim, a realização de treinamentos específicos e frequentes voltados à correta manipulação de alimentos é uma condição fundamental para a redução da contaminação da merenda escolar, pois, através destas ações, os manipuladores de alimentos se qualificam e se especializam nas boas práticas de higiene pessoal e alimentar (Campos et al., 2009).

Vasques e Madrona (2016) verificaram que a aplicação do checklist após a atividade de capacitação apresentou melhorias significativas nas condições higiênico-sanitárias do estabelecimento de alimentação, ressaltando que a capacitação da mão de obra é o fator mais importante do processo. Botega et al. (2010) relataram, ao realizar a capacitação em boas práticas para manipuladores em escolas da rede pública do Rio Grande do Sul, que para $71,8 \%$ dos participantes era a primeira capacitação sobre manipulação de alimentos, e que houve boa participação e aceitação, proporcionando discussões relevantes sobre as boas práticas no ambiente de trabalho.

Ao final de todas as atividades desenvolvidas, foi observado de forma mais clara as dificuldades percebidas pelos manipuladores de alimentos, através da compreensão do universo de trabalho destas, e das limitações impostas pela a falta de orientação e supervisão. As limitações que a escolas apresentam se relacionam à estrutura do prédio, orientação aos manipuladores e a falta de recursos para o melhor atendimento às boas práticas de manipulação.

\section{Considerações finais}

Com a realização desse estudo, foi observado diversas não conformidades nos espaços físicos, higiene de utensílios, e procedimentos por parte dos manipuladores de alimentos nas escolas públicas estudadas. Foram observados resultados satisfatórios, por grande parte dos manipuladores, na receptividade das informações quando levadas para prática, no entanto, houve uma certa resistência por outra parte dos manipuladores. Com 
isso, percebe-se a necessidade de serem realizadas ações e orientações sobre o cuidado sanitário dos alimentos de forma contínua, a fim de garantir a prevenção de doenças transmitidas por alimentos, para assim contribuir com um alimento seguro no ambiente escolar.

\section{Agradecimentos}

Às escolas através da direção, e aos participantes, pela gentileza e confiança ao se apresentarem voluntariamente nas atividades desenvolvidas dessa ação extensionista. Ao apoio institucional (reconhecimento desse projeto de extensão e concessão de bolsa extensão e cultura) concedido pela PróReitoria de Extensão e Cultura do Instituto Federal de Educação, Ciência e Tecnologia da Paraíba, e ao campus Sousa.

\section{Contribuição de cada autor}

Ambos os autores elaboram e executaram o projeto, e redigiram o texto aqui apresentado. J.M.G.O. e L.K.L. atuaram como coordenadoras dessa ação extensionista.

\section{Referências}

American Public Health Association (APHA). (2001). Committee on Microbiological for Foods. Compendium of methods for the microbiological examination of foods. 4. ed. Washington: American Public Health Association.

Botega, A. O., Gabbardo, F. G., \& Saccol, A. L. F. (2010). Capacitação em boas práticas para manipuladores da alimentação escolar da rede pública de ensino da região central do Rio Grande do Sul, Brasil. Disciplinarum Scientia: Ciência da Saúde, 11(1), 71-78.

Campos, A. K. C., Cardoso, M. S. C., Pinheiro, L. B. G., Ferreira, N. R., Azevedo, P. R. M., \& Stamford, T. L. M. (2009). Assessment of personal hygiene and practices of food handlers in municipal public schools of Natal, Brazil. Food Control, 20(9), 807-810.

Conselho Nacional de Segurança Alimentar e Nutricional (CONSEA) (2007). III Conferência Nacional de Segurança Alimentar e Nutricional: Por um desenvolvimento sustentável com soberania e segurança alimentar e nutricional. Brasília: CONSEA.

Dias, R. M. F., \& Santos, I. C. B. (2017). Aplicação das boas práticas em restaurantes e lanchonetes localizados em instituição de Ensino Superior de Salvador. Revista Higiene Alimentar, 31(270), 40-44.

Franco, B. D. G. M., \& Landgraf, M. (2008). Microbiologia dos Alimentos. São Paulo: Atheneu.

Gomes, N. A. A. A., Campos, M. R. H., \& Monego, E. T. (2012). Aspectos higiênico-sanitários no processo produtivo dos alimentos em escolas públicas do Estado de Goiás, Brasil. Revista de Nutrição, 25(4), 473-485.

Lopes, A. C. C., Pinto, H. R. F., Costa, D. C. I. O., Mascarenhas, R. J., \& Aquino, J. S. (2015). Avaliação das Boas Práticas em unidades de alimentação e nutrição de escolas públicas do município de Bayeux, PB, Brasil. Ciência \& Saúde Coletiva, 20(7), 2267-2275.

Machado, J. R., Marson, J. M., Oliveira, A. G. S., Silva, P. R., \& Terra, A. P. S. (2009). Avaliação microbiológica das mãos e fossas nasais de manipuladores de alimentos da unidade de alimentação e nutrição de um Hospital Universitário. Medicina (Ribeirão Preto), 42(4), 461-465.

Ministério da Saúde. (2018). Sistema de Vigilância em Saúde. Surtos de doenças transmitidas por alimentos no Brasil. [S. I.]: Ministério da Saúde. Recuperado de http://portalarquivos2.saude.gov.br/images/pdf/2018/ janeiro/17/Apresentacao-Surtos-DTA-2018.pdf

Moura, L. F. A. D., Lira, D. M. M. P., Moura, M. S., Barros, S. S. L. V., Lopes, T. S. P., Leopoldino, V. D., \& Moura, M. D. (2001). Apresentação do Programa Preventivo para Gestantes e Bebês. Jornal Brasileiro de Odontopediatria \& Odontologia do Bebê, 4(17), 10-14. 
Murray, P. R., Rosenthal K. S., \& Pfaller, M. A. (2014). Microbiologia Médica. 7. ed. Rio de Janeiro: Elsevier.

Pedrosa, A. C., Sylvestre S. H. Z., \& Fernandes, G. F. R. (2015). Avaliação microbiológica das mãos de manipuladores de alimentos de uma cozinha piloto do município de Pirangi-SP. International Journal of Medical Science and Clinical Inventions, 2(7), 1126-1134.

Pereira, W. B. B., \& Zanardo, V. P. S. (2020). Gestão de boas práticas em uma cantina escolar. Revista Vivências, 16(30), 193-200.

Silva E. V. C., Barata, F. T. M., Rocha, N. M. A., Barros, B. C. V., Silva, G. F., Silva, N. S., \& Silva, B. A. (2012a). Condições higiênico-sanitárias em escolas públicas do município de Castanhal - PA, após treinamento dos manipuladores de alimentos. Revista Higiene Alimentar, 26(212), 27-30.

Silva, C. B. G., \& Almeida, F. Q. A. (2011). Qualidade na produção de refeições de uma unidade de alimentação e nutrição (UAN). Revista Simbio-Logias, 4(6), 155-162.

Silva, L. P., Silva, S. C., \& Silva, R. Q. (2012b). Análise das boas práticas de fabricação de alimentos em cozinhas das escolas estaduais de Passos - MG: Da escolha do produto até o seu reaproveitamento. Ciência et Praxis, 5(9), 7-12.

Vasques, C. T., \& Madrona, G. S. (2016). Aplicação de checklist para avaliação da implantação das boas práticas em uma unidade de alimentação e nutrição. Revista Higiene Alimentar, 30(252), 53-58.

Vila, C. V. D., Silveira, J. T., \& Almeida, L. C. (2014). Condições higiênico-sanitárias de cozinhas de escolas públicas de Itaqui, Rio Grande do Sul, Brasil. Vigilância Sanitária Em Debate: Sociedade, Ciência \& Tecnologia, 2(2), 67-74.

Como citar este artigo:

Bezerra, N. M., Silva, K. R. R., Nésio, E. P. X., de Lima, L. K., \& de Oliveira, J. M.G. (2021). Avaliação das condições higiênico-sanitárias e treinamento de manipuladores de alimentos em instituições públicas no município de IpaumirimCE. Revista Brasileira de Extensão Universitária, 12(3), 339-348. https://periodicos.uffs.edu.br/index.php/RBEU/article/ view/11728/pdf 